\title{
Assessment of indoor and outdoor airborne fungi in an Educational, Research and Treatment Center
}

\author{
Nasrin Rostami, ${ }^{1}$ Hossien Alidadi, ${ }^{2}$ Hossein Zarrinfar, ${ }^{3,4}$ Pegah Salehi ${ }^{5}$ \\ ${ }^{1}$ Environmental Health Engineering, School of Health, Shiraz University of Medical Sciences, Shiraz; ${ }^{2}$ Environmental Health \\ Department, Health Sciences Research Center, School of Health, Mashhad University of Medical Sciences, Mashhad; ${ }^{3}$ Allergy \\ Research Center, School of Medicine, Mashhad University of Medical Sciences, Mashhad; ${ }^{4}$ Department of Parasitology and \\ Mycology, Ghaem Hospital, Faculty of Medicine, Mashhad University of Medical Sciences, Mashhad; ${ }^{5}$ Environmental Health \\ Engineering, School of Health, Esfahan University of Medical Sciences, Esfahan, Iran
}

\begin{abstract}
Hospital environments contain different types of microorganisms. Airborne fungi are one of these microbes and the major source of hospital indoor contamination that will be able to cause airborne fungal diseases. In the current study, the total count and diversity of the airborne filamentous and yeasts fungi were investigated in indoor and outdoor air of selective wards of Emam Reza Educational, Research and Treatment Center. This cross-sectional study was performed during the fall season. One hundred and ninety-two environmental samples of indoor and outdoor air from hematology, infectious diseases, Ear, Nose and Throat (ENT) and Neonatal Intensive Care Unit (NICU) wards were collected by open plate technique (on Sabouraud dextrose agar media) once a week. The cultures were then examined and evaluated according to macroscopic and microscopic examination criteria. In this study, 67 (62.03\%) of indoor samples and 81 (96.42\%) of outdoor samples were positive for fungi. The most isolated fungi were yeast species (17.12\%), Penicillium spp. (16.34\%), Alternaria spp. (14.39\%), Aspergillus niger (11.28\%), A. flavus (8.95\%), respectively. Almost all of the wards showed high rates of contamination by various fungi. However, the analysis of the data showed that indoor air of hematology ward had the highest fungal pollution. In contrast, the outdoor air of ENT had the highest fungal pollution. Thus, these results demonstrated that the cleansing and disinfection procedures in the hospital wards should be improved yet.
\end{abstract}

Correspondence: Hossein Zarrinfar, Department of Parasitology and Mycology, Ghaem Hospital, Mashhad University of Medical Sciences, Mashhad, Iran.

Tel.: +98.5138403141 - Fax: +98.5138547255.

E-mail: Zarrinfarh@mums.ac.ir

Key words: Hospital wards; bio-aerosol; airborne fungal; Mashhad.

Acknowledgments: we thank the staff at hematology, infectious diseases, ENT and NICU wards of Emam Reza Educational, Research and Treatment Center in Mashhad University of Medical Sciences (MUMS), for their help.

Funding: this study was financially supported by the Deputy of Research, MUMS, Mashhad, Iran (grant No. 901130).

Conflict of interest: the authors have no conflict of interest to declare.

Received for publication: 22 September 2015.

Accepted for publication: 4 April 2016.

This work is licensed under a Creative Commons Attribution NonCommercial 4.0 License (CC BY-NC 4.0).

(C) Copyright N. Rostami et al., 2017

Licensee PAGEPress, Italy

Italian Journal of Medicine 2017; 11:52-56

doi:10.4081/itjm.2016.663

\section{Introduction}

Physical, chemical and biological agents of the indoor and outdoor environment can affect the health and public welfare. ${ }^{1}$ Exposure to many microorganisms in indoor environments such as hospitals is unavoidable. ${ }^{2}$ Indoor air quality in hospitals is a concern because it contains a wide range of infectious airborne microorganisms that may cause hospital infections. ${ }^{3,4}$ According to some studies, hospital-acquired infections (HAI) or nosocomial infections are responsible for approximately $10 \%$ of the patients. ${ }^{5}$ Bio-aerosols are also known as causative agents of nosocomial infections for hospitalized patients, especially those requiring extended treatments and intensive care. ${ }^{5}$ Fungi and bacteria are the major types of bio-aerosol present in hospital environments. ${ }^{6}$

Fungal pollutant in indoor environment depends on many factors such as: temperature, moisture, ventilation, organic matter present in building materials. Also, outdoor fungal spores ${ }^{6}$ may be transmitted through visitors, patients and air conditioning. ${ }^{2} \mathrm{~A}$ large number of studies have showed that various percentages of hospital infections were caused by fungi, such as: Candida albicans and diverse species of Aspergillus, Cladosporium, and Penicillium. ${ }^{7}$ The most frequent species of 
fungal bio-aerosols isolated from hospital indoor air were A. flavus, Penicillium spp., Fusarium spp., C. albicans and Alternaria spp.. ${ }^{8}$ The evaluation of density and diversity of bio-aerosols in the hospital can be a good indicator of the cleanliness of these environments. ${ }^{4}$ Therefore, assessment of indoor air quality in hospitals will help to find the origin of the infection, as a result, can help in reducing fungal nosocomial infections rates. The objective of this study was to assess the level of fungal contamination of indoor and outdoor air, and the most common types of fungi in selective wards of Emam Reza Educational, Research and Treatment Center in Mashhad in 2012.

\section{Materials and Methods}

\section{Study area}

This cross-sectional study was performed during the fall in Emam Reza Educational, Research and Treatment Center in Mashhad city in Iran. The wards of Hematology, Infectious Diseases, Ear, Nose and Throat (ENT) and Neonatal Intensive Care Unit (NICU) wards, due to the type of hospitalized patients and their increased susceptibility to respiratory fungal infections, were selected. The indoor and outdoor air samples were collected at weekly intervals.

\section{Air sampling procedure}

The air samples were collected by passive sampling method (open plate method). ${ }^{9}$ This method uses settle plates, which are standard Petri dishes containing culture media of Sabouraud dextrose agar with chloramphenicol. They were exposed to the air for a given period of time in order to collect biological par- ticles for the sediment quality evaluation. ${ }^{10}$ The Petri dishes were put at a height of $100-150 \mathrm{~cm}$ above the ground level during sampling for 20 min. ${ }^{11}$ Finally over a three-month period, 108 plates were exposed to selective wards indoor air and 84 plates to outdoor air and samples were collected.

After exposure, the plates were transported in a clean container to the laboratory for microbiological examination. The plates were placed in incubator at $32^{\circ} \mathrm{C}$ for $7-10$ days. Then, the fungal colonies were identified according to morphological (color, texture, shape, diameter appearance of colony) and microscopic characteristics (presence of specific reproductive structures, presence/absence of conidia and their size, shape and structure of conidia, septation in mycelium). The lactophenol cotton blue wet mount preparation was used for staining and observing the fungi in direct examination. ${ }^{12}$

\section{Statistical analysis}

The data were analyzed using SPSS software version 16 and Chi-square test such as simple mean value, percentage and test of significance. ${ }^{8}$

\section{Results}

Almost all of the wards were polluted by various fungi. In this study, 67 (62.03\%) of indoor samples and $81(96.42 \%)$ of outdoor samples were positive for fungi that 306 fungal colonies were isolated. The most isolated fungi were yeast species $(17.12 \%)$, Penicillium spp. (16.34\%), Alternaria spp. (14.39\%), A. niger (11.28\%), A. flavus (8.95\%) and the lowest number were Geotrichum spp. (1.17\%), Rhizopus spp. $(1.56 \%)$, A. terreus $(2.33 \%)$, respectively (Figure 1$)$.

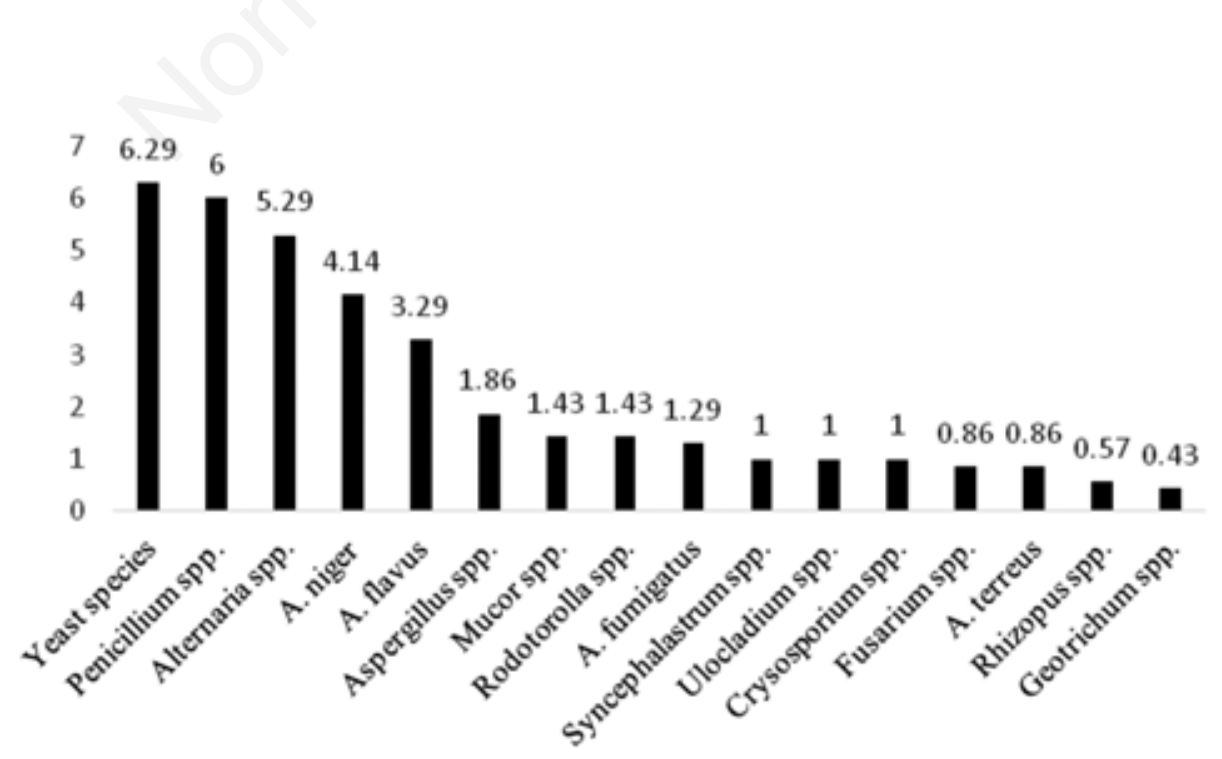

Figure 1. Mean of isolated fungi in indoor and outdoor air of selective wards of Emam Reza Educational, Research and Treatment Center in Mashhad. 
The dominant fungi isolated from the indoor air of different wards included: Penicillum spp. and yeast species from ENT ward; yeast species and A. flavus from hematology ward; Penicillum spp. and yeast species from infection ward; A. niger and Chrysosporium spp. from NICU ward. The indoor air of hematology had the highest and ENT ward had the lowest fungal pollution. In contrast, the outdoor air of ENT ward had the highest and infection wards had the lowest fungal pollution (Table 1).

The mean of different fungal genera isolated from indoor and outdoor plates were 2.58 and 7.93 fungus/plate, respectively. It indicates a significant dif- ference between indoor and outdoor air quality.

The most identified fungi in indoor air of wards were yeast species, Penicillium spp., A. flavus and in outdoor air of wards were Alternaria spp., Penicillium spp., A. niger, respectively (Table 2).

\section{Discussion and Conclusions}

The present study showed that all wards of the hospital including hematology, infectious diseases, ENT and NICU were contaminated with high rates of different genus and species of fungi. Airborne fungal

Table 1. Concentration of fungal population in indoor and outdoor air of different wards of selective wards of Emam Reza Educational, Research and Treatment Center in Mashhad.

\begin{tabular}{|c|c|c|c|}
\hline Place of sampling & CFU & Mean & Standard deviation \\
\hline Indoor air of Hematology ward & 33 & 3.78 & 3.456 \\
\hline Indoor air of Infection Diseases ward & 20 & 2.22 & 1.202 \\
\hline Indoor air of NICU ward & 21 & 2.33 & 1.414 \\
\hline Indoor air of ENT ward & 18 & 2 & 1 \\
\hline Outdoor air of Hematology and Infection Diseases wards* & 55 & 6.11 & 2.934 \\
\hline Outdoor air of NICU ward & 73 & 8.11 & 3.723 \\
\hline Outdoor air of ENT ward & 86 & 9.56 & 3.877 \\
\hline $\mathrm{F}=11.8$ & & $\mathrm{P}<0.001$ & \\
\hline
\end{tabular}

*Hematology and Infection Diseases wards were in one department. CFU, Colony Forming Unit; ENT, Ear, Nose and Throat; NICU, Neonatal Intensive Care Unit.

Table 2. Percentage of detected fungi in indoor and outdoor air of different wards of selective wards of Emam Reza Educational, Research and Treatment Center in Mashhad.

\begin{tabular}{|c|c|c|}
\hline Fungus & Fungi isolated in indoor air (\%) & Fungi isolated in outdoor air (\%) \\
\hline A. niger & 5 & 14 \\
\hline A. fumigatus & 1 & 4 \\
\hline A. flavus & 9 & 9 \\
\hline A. terreus & 3 & 2 \\
\hline Aspergillus spp. & 3 & 6 \\
\hline Alternaria spp. & 5 & 18 \\
\hline Geotrichum spp. & 3 & 1 \\
\hline Penicillium spp. & 17 & 16 \\
\hline Rhizopus spp. & 3 & 1 \\
\hline Mucor spp. & 8 & 2 \\
\hline Syncephalastrum spp. & 3 & 3 \\
\hline Ulocladium spp. & 3 & 3 \\
\hline Fusarium spp. & 1 & 3 \\
\hline Chrysosporium spp. & 5 & 2 \\
\hline Yeast species & 28 & 12 \\
\hline
\end{tabular}


spores are very important agents in nosocomial infection and respiratory diseases and their effect on human health linked with their genera, species and concentrations in air., ${ }^{9,11}$ Many studies have showed the relationship between HAI or nosocomial infection and the existence of microorganisms in hospital environments, including in the air of wards. ${ }^{3,8}$ As an example, HAI rates have ranged from $1 \%$ in parts of Europe and North America to more than $40 \%$ in certain parts of Asia, Latin America and Sub-Saharan Africa. ${ }^{13}$

During the past two decades, incidence of fungal diseases including invasive aspergillosis has increased in hospitalized patients, who are particularly immunosuppressed patients. ${ }^{11}$ Therefore, assessment of airborne fungal spores is important and can help decrease HAI rates. ${ }^{14}$ In this study, some fungi isolated included A. flavus, Penicillium spp., Fusarium spp. and Alternaria spp. which were similar to what has been reported in previous researches. ${ }^{8}$ According to the results obtained in this study, the most frequent fungal populations recovered from both indoor and outdoor air were yeast species, Penicillium spp., Alternaria spp., $A$. niger and $A$. flavus, respectively. In addition, the fungal genera distribution was similar to previous studies, for example Sonmez et al. have reported the most common genus were Penicillium, Alternaria and Aspergillus. ${ }^{15}$ Other studies also showed that the most frequently isolated genera were Aspergillus, Penicillium, Alternaria ${ }^{8,9}$ It seems that different geographic locations can influence the dominant fungal agents. For examples, A. flavus isolated frequently from saprophytic fungal infections in Iran. ${ }^{16-18}$ In a previous study in Iran, the major genera in the kidney transplant unit were Penicillium, Cladosporium, Aspergillus and Alternaria. ${ }^{11}$ In our study, A. fumigatus was the third most prevalent Aspergillus species, after $A$. niger and $A$. flavus, isolated from the air hospital wards, while in the two similar studies in Iran did not isolate any $A$. fumigatus from the hospitals. However, our results were in accordance with those reported by Panagopoulou et $a l$. in Greece, he achieved similar results to the results of current study. ${ }^{19}$ According to the data obtained in our study, indoor air of ENT ward had the lowest fungal bioaerosols. This could be due to the appropriate cleaning and disinfection procedures, air conditioning system and ultraviolet light application in this ward. ${ }^{4}$ The microbiological quality of the air in operating room such as ENT is an important parameter for controlling and preventing nosocomial infections and surgical wound infections. ${ }^{10}$ Fungal pollution of indoor environments depends on several factors, such as moisture, ventilation, temperature, organic matter present in building materials and the outdoor airborne fungal spores load. ${ }^{6}$ The effect of the outdoor fungal flora on the indoor has been known, however, it is a proven fact that the indoor airborne fungi, regardless of the type, has two sources: the outdoor air and the indoor fungal colonization. Such colonization originates mainly in every wet, dark and area with air ventilated unfavorable. ${ }^{11}$ Our results showed a significant difference between indoor and outdoor air quality. The rate of isolation fungi outdoors was higher than indoors. This observation is compatible with the hypothesis that outdoor air has a considerable influence on indoor air quality. ${ }^{9}$ In this study, the data showed that $62.03 \%$ of indoor air samples were contaminated with airborne fungi, that this may be due to opening doors and windows and construction and demolition at the hospital during the study, high number of patients' visitors. As shown by previous studies, a significant increase in fungal counts during building activities was observed. The risk of fungal contamination and the HAI rates can be reduced by using appropriate air filtration devices where construction activities exist in the area of hospitals. ${ }^{20}$ In addition, increasing use of air conditioning and air filtration systems in hospital without appropriate engineering controls to maintain temperature and relative humidity at favorable levels may facilitate microbial growth. ${ }^{3}$ Although the current study was performed in fall season, there is not accurate information on fluctuations in bioaerosol loads with season changing. On the other hand, hospital wards design standards are an important parameter, where incorrect design may lead to low indoor air quality, increase in airborne microorganisms particularly airborne fungus. ${ }^{3}$ Our results showed that almost all of the wards were polluted by various fungi. Regular surveillance and stringent measures including air disinfection system, ventilation systems, using the HEPA filters for high-risk ward, closing the windows, control entry and exit doors, control or totally eliminate of flower taken by the patients' visitors are necessary to reduce mold spores.

\section{References}

1. Ekhaise F, Ighosewe O, Ajakpovi O. Hospital indoor airborne microflora in private and government owned hospitals in Benin City, Nigeria. World J Med Sci 2008;3: 19-23.

2. Park D-U, Yeom J-K, Lee WJ, Lee K-M. Assessment of the levels of airborne bacteria, gram-negative bacteria, and fungi in hospital lobbies. Environ Res Public Health 2013;2013:541-55.

3. Sudharsanam S, Swaminathan S, Ramalingam A, et al. Characterization of indoor bioaerosols from a hospital ward in a tropical setting. Afr Health Sci 2012;12:217-25.

4. Hoseinzadeh E, Samarghandie MR, Ghiasian SA, et al. Evaluation of bioaerosols in five educational hospitals wards air in Hamedan, during 2011-2012. Jundishapur J Microbiol 2013;6:1-8.

5. Huang P-Y, Shi Z-Y, Chen C-H, et al. Airborne and surface-bound microbial contamination in two intensive care units of a medical center in Central Taiwan. Aerosol Air Qual Res 2013;13:1060-9. 
6. Sautour M, Sixt N, Dalle F, et al. Profiles and seasonal distribution of airborne fungi in indoor and outdoor environments at a French hospital. Sci Total Environ 2009;407:3766-71.

7. Azimi F, Naddafi K, Nabizadeh R, et al. Fungal air quality in hospital rooms: a case study in Tehran, Iran. J Environ Health Sci Engine 2013;11:30.

8. Awosika S, Olajubu F, Amusa N. Microbiological assessment of indoor air of a teaching hospital in Nigeria. Asian Pacif J Trop Biomed 2012;2:465-8.

9. Uzochukwu OV, Nkpouto U. Airborne fungi in the indoor and outdoor environments of a higher institution in Nigeria. Int J Adv Biol Res 2013;3:9-12.

10. Napoli C, Tafuri S, Montenegro L, et al. Air sampling methods to evaluate microbial contamination in operating theatres: results of a comparative study in an orthopaedics department. J Hospital Infect 2012;80:128-32.

11. Afshari MA, Riazipour M, Kachuei R, et al. A qualitative and quantitative study monitoring airborne fungal flora in the Kidney Transplant Unit. Nephro-Urol Month 2013;5:736-40.

12. Singh G, Nautiyal O, Singh AK, Bedi MK. Assessment of airborne microflora in the Academic Institute of Dehradun. Int J Res Appl Nat Social Sci 2013;1:9-18.

13. Saxena P, Mani RK. Preventing hospital acquired infections: a challenge we must accept. Indian J Crit Care Med 2014;18:125-6.

14. Saadoun I, Tayyar IAA, Elnasser Z. Concentrations of airborne fungal contamination in the medical surgery operation theaters (OT) of different hospitals in Northern Jordan. Jordan J Biol Sci 2008;1:181-4.

15. Sonmez E, Ozdemir HM, Cem EM, et al. Microbiological detection of bacteria and fungi in the autopsy room. Romanian J Legal Med 2011;19:33-44.

16. Zarrinfar H, Saber S, Kordbacheh P, et al. Mycological microscopic and culture examination of 400 bronchoalveolar lavage (BAL) samples. Iran J Public Health 2012;41:70-6.

17. Zarrinfar H, Makimura K, Satoh K, et al. Incidence of pulmonary aspergillosis and correlation of conventional diagnostic methods with nested PCR and real-time PCR assay using BAL fluid in intensive care unit patients. J Clin Lab Anal 2013;27:181-5.

18. Zarrinfar H, Mirhendi H, Makimura K, et al. Use of mycological, nested PCR, and real-time PCR methods on BAL fluids for detection of Aspergillus fumigatus and A. flavus in solid organ transplant recipients. Mycopathologia 2013;176:377-85.

19. Sepahvand A, Shams-Ghahfarokhi M, Allameh A, Razzaghi-Abyaneh M. Diversity and distribution patterns of airborne microfungi in indoor and outdoor hospital environments in Khorramabad, Southwest Iran. Jundishapur J Microbiol 2013;6:186-92.

20. Sautour M, Sixt N, Dalle F, et al. Prospective survey of indoor fungal contamination in hospital during a period of building construction. J Hospital Infect 2007;2007: 367-73. 